\title{
Unstable cervical spine; is it still a contraindication for percutaneous tracheostomy as thought before?
}

\author{
Amila Jayasinghe $^{1^{*}}$, Ravi Weerakoon ${ }^{2}$, Udaya Karunarathne ${ }^{2}$ \\ Senior Registrar in Anaesthesia ${ }^{1 *}$, Consultant Anaesthetist ${ }^{2}$, Neurosurgical Intensive Care Unit, \\ TH Kandy.
}

\begin{abstract}
We report a 28 year old male with an unstable acute traumatic cervical spinal cord injury (SCI) due to anterior spondylolisthesis of $\mathrm{C} 6$ over $\mathrm{C} 7$ vertebra and facet joint dislocation of C6-C7 who underwent a successful bronchoscopy guided percutaneous tracheostomy (PCT) on day 6. Cervical SCI demands tracheostomy in majority but SCI with unstable cervical spine itself makes tracheostomy a challenging procedure. Even though cervical SCI was considered a relative contraindication for PCT, it can be safely performed in neutral neck position. Addition of ultrasound scanning of neck and bronchoscopic guidance further increases its safety profile. In cervical SCI early tracheostomy may be more effective than late.
\end{abstract}

\section{Case History}

A 28 year old man sustained an acute cervical SCI following a high speed road traffic accident. CT cervical spine showed anterior spondylolisthesis of C6 over $\mathrm{C} 7$ vertebra and facet joint dislocation of C6-C7. He had evidence of cervical spinal cord damage as he had mechanical respiratory failure, hypotension with bradycardia and partial muscle weakness below T1 level. According to American Spinal Injury Association (ASIA) Impairment Scale he fell into category "C". Since he was predicted to have mechanical ventilation for a longer duration a tracheostomy was decided on day 6 of injury. Being a unit performing percutaneous tracheostomy (PCT) for more than 4 years we decided to go ahead with PCT. Informed consent was taken. Patient was on minimal ventilatory support with $40 \%$ oxygen. There was no coagulopathy. He was of normal built and had no anatomical abnormality of the neck. Patient was positioned supine with neck in neutral position with the skull callipers which were inserted since admission. Bedside ultrasound scan of the neck did

*Correspondence: Amila Jayasinghe

E mail: amila.j.84@gmail.com

https://orcid.org/0000-0002-0130-6971

Received: $13 / 06 / 2020$

Accepted: $27 / 06 / 2020$

DOI: http:/doi.org/10.4038/slja.v28i2.8615 not reveal any large anterior vessels or large thyroid which can lead to bleeding. Thyroid cartilage, cricoid cartilage and sternal notch were identified, and entry point was marked between the latter two. He was preoxygenated, sedated and paralysed. It was a bronchoscopy guided procedure using a single dilator system and it was performed by the most experienced anaesthetist to minimize complications. We did not note any complications and patient did not show any worsening of neurological signs after the procedure.

\section{Discussion}

Acute traumatic SCI occurs in about 15-35 per million population. ${ }^{1}$ It is interesting to see that acute cervical SCI demands tracheostomy due to several reasons but presence of an unstable cervical spine makes it highly challenging. These patients require prolong mechanical ventilation due to low lung capacity as respiratory muscles are denervated, impaired clearance of tracheobronchial secretions and high incidence of pneumonia. ${ }^{1}$ While enabling prolonged ventilatory support, tracheostomy will also facilitate weaning from mechanical ventilation as it decreases anatomical dead space hence reducing work of breathing and airway resistance. It also reduces the need for sedation and facilitates tracheo-bronchial toileting. This allows better oral care and patient finds it more comfortable. ${ }^{2}$ Tracheostomy is reported to lower the mortality rate and to prevent complications of a prolonged orotracheal intubation. ${ }^{3}$

Even though tracheostomy was traditionally done surgically; percutaneous tracheostomy (PCT) has 
become more popular over the past decades after its first description by Ciaglia in $1985 .{ }^{2}$ Over the time, several modifications were done to improve this technique and it is getting popular faster. Today there are PCT kits which have either a special dilating forcepsor a single-tapered dilator. The latter being more efficient and easier, it is widely used. $^{2,3}$

There are many advantages of PCT in cervical SCI. It is less invasive, and it can be performed as a bedside procedure.Transporting a patient with an unstable cervical spine could exacerbate cord injury during transport as well as during transfer of patient from beds to trolleys. Since they are cardiovascularly unstable it adds more to the risk. These can be eliminated by PCT as it is done at point of care. Since the surgeons had decided for cervical spinal fixation to be done later, performing a surgical tracheostomy during the spinal fixation was also not an option in our case. The most important fact which makes PCT a reality in acute cervical SCI is, it can be conducted with the neck in a neutral position. ${ }^{3,4,5}$ The presence of facet joint dislocation with anterior spondylolisthesis of C6 over $\mathrm{C} 7$ with some degree of rotation instability of $\mathrm{C} 6$ over $\mathrm{C} 7$ is an unstable injury. Our patient's neck extension, flexion as well as rotation can bring about serious complications. Hence, we decided to keep head in neutral position with skull traction in place. Sometimes there may be difficulty in identifying landmarks clearly in a small percentage when neck is not extended. ${ }^{5}$ This can be overcome by concomitant use of neck ultrasound scanning. PCT has a low rate of complications such as bleeding and wound infection probably due to minimal tissue injury. ${ }^{3}$ It is technically easy to perform and has a fast learning curve. ${ }^{3}$ Acute cervical SCI is considered a relative contraindication for PCT because of inability to extend the neck, the presence of surgical scar of open reduction or external fixation devices such as skull traction or Philadelphia collar. However, PCT can be done safely in acute unstable cervical spinal injury by an experienced team as the advantages of PCT became more apparent. ${ }^{5,6,7}$ Rrisks associated with PCT such as false tract formation and posterior tracheal wall perforation can be further reduced by addition of bronchoscopy guidance. Use of ultrasound in critical care further facilitates PCT by enabling identification of landmarks as well as presence of thyroid tissue and vessels on the pathway with can cause potential bleeding. ${ }^{3}$

A retrospective study has evaluated the benefits and safety of early versus late tracheostomy in mechanically ventilated traumatic SCI patients. It showed that early placement before day 7 of mechanical ventilation shortens the duration of mechanical ventilation, reduces ICU stay and complications of orotracheal intubation like tracheal stenosis. However, they found no benefit in rates of ventilator-associated pneumonia and mortality. ${ }^{8}$ Favourable effects in early tracheostomy are demonstrated in other studies as well. ${ }^{1,5}$

\section{Conclusion}

PCT is a demanding but challenging procedure in acute cervical SCI patients. It can be safely performed if expertise is available without further cord damage in neutral neck position despite the presence of an unstable cervical spine. There are advantages of early tracheostomy compared to late in SCI. Given the many advantages of PCT, it should be considered the first choice for tracheostomy and acute cervical spinal injury is no more a contraindication for PCT.

\section{References}

1. Bonner S, Smith C. Initial management of acute spinal cord injury Continuing Education in Anaesthesia Critical Care \& Pain 2013; 13(6): 224231 . https://doi.org/10.1093/bjaceaccp/mkt021

2. Ansari MAA, Hijazi MH.Clinical review: Percutaneous dilatational tracheostomy. Critical Care200510:202

3. Ganuza J, Oliviero A.Tracheostomy in Spinal Cord Injured Patients.Transl Med UniSa 2011; 1: 151172 .

4. Mayberry JC, Wu IC, Goldman RK et al. Cervical spine clearance and neck extension during percutaneous tracheostomy in trauma patients. Crit Care Med. 2000 ;28(10):3436-40. https://doi.org/10.1097/00003246-200010000$\underline{00011}$

PMid:1 1057798

5. Nun AB, Orlovsky M, Best LA. Institutional report - Thoracic general Percutaneous tracheostomy in patients with cervical spine fractures-feasible and safe. Interactive CardioVascular and Thoracic Surgery 2006; 5: 427-429. 
Jayasinghe et al. Sri Lankan Journal of Anaesthesiology: 28(2):165-167 (2020)

https://doi.org/10.1510/icvts.2005.125690

PMid:17670607

6. O'Keeffe T, Goldman RK, Mayberry JC et al. Tracheostomy after anterior cervical spine fixation.

J Trauma. 2004;57(4):855-60.

https://doi.org/10.1097/01.TA.0000083006.48501.

$\underline{\mathrm{B} 2}$

PMid:15514542

7. Romero J, Vare A, Gamgarrutta $\mathrm{C}$ et al. Tracheostomy timing in traumatic spinal cord injury. Eur Spine J. 2009; 18(10): 1452-1457. https://doi.org/10.1007/s00586-009-1097-3

PMid:19655178 PMCid:PMC2899388

8. Ganuza J, Forcada AG, Gambarrutta C et al. Effect of technique and timing of tracheostomy in patients with acute traumatic spinal cord injury undergoing mechanical ventilation. J Spinal Cord Med. 2011; 34(1): 76-84.

https://doi.org/10.1179/107902610X128862610918

$\underline{75}$

PMid:21528630 PMCid:PMC3066483 